\title{
Moduli of quadrilaterals and extremal quasiconformal extensions of quasisymmetric functions
}

\author{
Shengjian $\mathrm{Wu}^{*}$
}

\begin{abstract}
We establish a relationship between Strebel boundary dilatation of a quasisymmetric function of the unit circle and indicated by the change in the module of the quadrilaterals with vertices on the circle. By using general theory of universal Teichmüller space, we show that there are many quasisymmetric functions of the circle have the property that the smallest dilatation for a quasiconformal extension of a quasisymmetric function of the unit circle is larger than indicated by the change in the module of quadrilaterals with vertices on the circle.
\end{abstract}

Mathematics Subject Classification (1991). Primary 32G15; Secondary 30C60; 30C75.

Keywords. Boundary dilatation, quasisymmetric function, quasiconformal mapping, Strebel point.

\section{$\S 1$. Introduction}

In this paper, the following notation will be used. $C=$ the finite complex plane; $\Delta=\{z \in C ;|z|<1\} ; \Gamma=\partial \Delta$ (boundary of $\Delta$ ); $\bar{\Delta}=\Delta \cup \Gamma ; \Delta_{r}=\{z ; r<|z|<1\}$, where $0<r<1 ; H=$ the upper half plane; $R=$ the real line in $C$.

Let $f: \Gamma \rightarrow \Gamma$ be a sense-preserving homeomorphism. We say $f$ is quasisymmetric if there exists a quasiconformal mapping $\tilde{f}: \bar{\Delta} \rightarrow \bar{\Delta}$ such that $\left.\tilde{f}\right|_{\Gamma}=f$. Let $z_{1}, z_{2}, z_{3}$ and $z_{4}$ be four points on $\Gamma$ following each other in the positive (anticlockwise) direction. Then they determine an unique topological quadrilateral with domain $\Delta$ and vertices $z_{1}, z_{2}, z_{3}$ and $z_{4}$ which we denote by $Q=Q\left(z_{1}, z_{2}, z_{3}, z_{4}\right)$. We will denote the conformal module of $Q$ by $M(Q)$. The function $f$ maps $Q$ to a quadrilateral $f(Q)=Q\left(f\left(z_{1}\right), f\left(z_{2}\right), f\left(z_{3}\right), f\left(z_{4}\right)\right)$. Now assume $f$ is quasisymmetric.It follows from the theory of quasiconformal mappings that for any

*This work was partially supported by grants from the NSF of China and Göran Gustafsson Fundation. 
quasiconformal extension $\tilde{f}$ and any quadrilateral $Q$ whose domain is $\Delta$

$$
\frac{1}{K(\tilde{f})} \leq \frac{M(f(Q))}{M(Q)} \leq K(\tilde{f})
$$

Thus the following number

$$
K_{0}=K_{0}(f)=\sup _{Q}\left\{\frac{M(f(Q))}{M(Q)} ; Q \text { is a quadrilateral with domain } \Delta\right\}
$$

is finite.

We distinguish two cases for $K_{0}(f)$. If there exists a non-degenerated quadrilateral $Q$ such that $K_{0}(f)=\frac{M(f(Q))}{M(Q)}$, we will use $K_{0}^{q}(f)$ instead of $K_{0}(f)$. We will use $K_{0}^{d}(f)$ instead of $K_{0}(f)$, if there is no non-degenerated quadrilateral such that $K_{0}(f)=\frac{M(f(Q))}{M(Q)}$.

We define

$$
K_{1}(f)=\inf \{K ; f \text { has a K-quasiconformal extension to a selfmap of } \bar{\Delta}\}
$$

where the infinum is taken for all quasiconformal extensions $\tilde{f}$ of $f$ to $\Delta$.

The following notations of boundary dilatation and local dilatation were introduced by Strebel (cf. [10] and [11]):

$$
H(f)=\inf \left\{K ; f \text { has a K-quasiconformal extension } \tilde{f}_{r} \text { to } \Delta_{r}\right\},
$$

where the infimum is taken for all quasiconformal extensions $\tilde{f}_{r}$ of $f$ to $\Delta_{r}$ and for all $r(0<r<1)$.

For a point $\xi$ on $\Gamma$

$$
H_{\xi}(f)=\inf \left\{K ; f \text { has a K-quasiconformal extension } \tilde{f}_{\varepsilon} \text { to } U_{\xi}(\varepsilon)\right\},
$$

where the infimum is taken for all quasiconformal extensions $\tilde{f}_{\varepsilon}$ of $f$ to a neighborhood of $U_{\xi}(\varepsilon)$ and all neighborhoods $U_{\xi}(\varepsilon)$ of $\xi$.

Obviously we have

$$
K_{0}(f) \leq K_{1}(f)
$$

and

$$
H(f) \leq K_{1}(f) .
$$

Fehlmann proved the following important result (cf. [4]):

$$
H(f)=\max _{\xi} H_{\xi}(f)
$$


In this paper we shall first establish a relationship between $K_{0}(f)$ and $H(f)$. To be precise, we shall prove the following result.

Theorem 1. Let $f: \Gamma \rightarrow \Gamma$ be a quasisymmetric function. Then either $K_{0}(f)=$ $K_{0}^{q}(f)$ or $K_{0}^{d}(f) \leq H(f)$.

We note that in [12] it was conjectured that $K_{0}(f)=K_{1}(f)$ for all quasisymmetric functions $f$. Anderson and Hinkkanen disproved this conjecture by giving concrete examples of a family of affine stretch mappings of some parallelograms (cf. [1]). We shall use the results in this paper to give a simpler proof of the result in $[1]$.

We shall use Theorem 1 and the theory of universal Teichmüller space to show many quasisymmetric functions $f$ have the property that $K_{0}(f)<K_{1}(f)$.

Let us recall some notations in Teichmüller theory. Let $Q S(\Gamma)$ be the full set of quasisymmetric functions of $\Gamma$ and let $\operatorname{Möb}(\Gamma)$ be the group of Möbius transformations mapping $\Gamma$ to itself. Then the right coset space $Q S(\Gamma) / \operatorname{Möb}(\Gamma)$ is the universal Teichmüller space $\mathcal{T}$. For any $f \in Q S(\Gamma)$, let $[f] \in \mathcal{T}$ be the Teichmüller class containing $f$.

Note that if $f \in Q S(\Gamma)$ and $g \in \operatorname{Möb}(\Gamma)$, then the quantities of $K_{0}(g \circ f)$, $K_{1}(g \circ f)$ and $H(g \circ f)$ are the same as $K_{0}(f), K_{1}(f)$ and $H(f)$, respectively. In other words, they are determined by the Teichmüller class $[f]$. Therefore we can define $K_{0}([f])=K_{0}(f)$. Similarly we can define $K_{1}([f])$ and $H([f])$ (but not $H_{\xi}([f])$.

In a recent paper, Earle and Li studied the geometry of infinite dimensional Teichmüller spaces (cf.[3]). Following them we call a point $[f] \in \mathcal{T}$ is a Strebel point if $H([f])<K_{1}([f])$. Let $\mathcal{T}_{S}$ be the set of all Strebel points in $\mathcal{T}$ and $\mathcal{T}_{N}=\mathcal{T} \backslash \mathcal{T}_{S}$.

The case $K_{0}^{q}(f)=K_{1}(f)$ in Theorem 1 is easy to describe and there are not "many" points in $\mathcal{T}$ such that the case holds.

Theorem 2. Let $U=\left\{[f] \in \mathcal{T} ; K_{0}^{q}(f)=K_{1}(f)\right\}$. Then $U$ depends on two real parameters and $U \subset \mathcal{T}_{S}$.

If $K_{0}([f])=K_{1}([f])$ and $[f] \notin U$, then $K_{0}([f])=H([f])$. Consequently $K_{1}([f])=H([f])$, that is, $[f]$ is a non-Strebel point. Theorem 2 tells us that if $K_{0}([f])=K_{1}([f])$, then $K_{0}^{q}([f])=K_{1}([f])$ and $K_{0}^{d}([f])=K_{1}([f])$ cannot hold simultaneously. Thus we have the following result.

Theorem 3. For every point $[f] \in \mathcal{T}_{S} \backslash U,[f]$ has the property that $K_{0}([f])<$ $K_{1}([f])$.

Recall that for any two points $\left[f_{j}\right] \in \mathcal{T},(j=1,2)$, the Teichmüller distance 
between them is defined by

$$
d\left(\left[f_{1}\right],\left[f_{2}\right]\right)=\frac{1}{2} \log K_{1}\left(f_{1} \circ f_{2}^{-1}\right) .
$$

From the definition of Strebel point, it is easy to see that, in the topology induced by the Teichmüller metric, $\mathcal{T}_{S}$ is an open set in $\mathcal{T}$. Since $U$ depends only on two real parameters and $\mathcal{T}$ is infinite dimensional, Theorem 3 tells us that many quasisymmetric functions $f$ have the property that $K_{0}(f)<K_{1}(f)$.

To give a concrete example, let us denote $\mathcal{T}_{0} \subset \mathcal{T}$ to be the set of all $[f] \in \mathcal{T}$ such that $H([f])=1$. Then $\mathcal{T}_{0}$ is also an infinite dimensional complex Banach manifold (cf. [6] and [7]). We can prove the following result.

Theorem 4. Every $[f] \in \mathcal{T}_{0} \backslash\{[i d]\}$ has the property that $K_{0}([f])<K_{1}([f])$.

Problem. Is it true that every non-Strebel point $[f]$ has the property that $K_{0}([f])$ $=K_{1}([f])$ ?

We shall prove the results above in the next subsections and in the final section we will discuss affine stretch mappings and give a simpler proof of the main result in $[1]$.

This work was partially done when the author was visiting the Department of Mathematics, Royal Institute of Technology at Stockholm. He wishes to thank Prof. M. Benedicks for his invitation, Göran Gustafsson Fundation for the financial support and the Department of Mathematics for its hospitality. He also wishes to thank Prof. J. Anderson for drawing his attention to the paper [1] and Prof. L. Carleson for useful discussions.

\section{$\S$ 2. Proof of Theorem 1}

In this section, we will prove the main result Theorem 1. Let $f \in Q S(\Gamma)$ and $K_{0}^{d}(f)=K_{0}(f)$. We shall prove that $K_{0}^{d}(f) \leq H(f)$.

Assume that $\left\{Q_{n}\right\}$ is a sequence of qudrilaterals with domain $\Delta$ such that

$$
\lim _{n \rightarrow \infty} \frac{M\left(f\left(Q_{n}\right)\right)}{M\left(Q_{n}\right)}=K_{0}^{d}(f)=K_{0}(f) .
$$

By passing to subsequences, if necessary, we may assume that the vertices $z_{j, n}(1 \leq$ $j \leq 4)$ of $Q_{n}$ tend to limit points $z_{j} \in \Gamma$ for $1 \leq j \leq 4$ as $n \rightarrow \infty$ and that at least two of the points $z_{j}$ coincide. Otherwise we will have $K_{0}^{q}(f)=K_{0}(f)$.

As in [1], there are the following four possibilities, up to permutations.

(1) $z_{1}=z_{2}$ while $z_{1}, z_{3}$ and $z_{4}$ are distinct;

(2) $z_{1}=z_{2} \neq z_{3}=z_{4}$ 
(3) $z_{1}=z_{2}=z_{3} \neq z_{4}$

(4) $z_{1}=z_{2}=z_{3}=z_{4}$.

In the proof it will be clear that if

$$
\lim _{n \rightarrow \infty} \frac{M\left(f\left(Q_{n}\right)\right)}{M(Q)}=\frac{1}{K_{0}(f)},
$$

we also have $K_{0}^{d}(f) \leq H(f)$.

We shall treat each case seperately.

\section{Case (1). Two points degeneracy}

Set

$$
\phi_{n}(z)=\frac{\left(z-z_{3, n}\right)\left(z_{4, n}-z_{2, n}\right)}{\left(z-z_{2, n}\right)\left(z_{4, n}-z_{3, n}\right)} .
$$

Then $\phi_{n}$ map $\Delta$ conformally onto $H$ taking $z_{1, n}, z_{2, n}, z_{3, n}, z_{4, n}$ onto $a_{n}, \infty, 0,1$ respectively. We have $1<a_{n}<\infty$ and $a_{n} \rightarrow \infty$ as $n \rightarrow \infty$. Similarly we set $w_{j, n}=f\left(z_{j, n}\right)$ for $1 \leq j \leq 4$ and

$$
\tilde{\phi}_{n}(w)=\frac{\left(w-w_{3, n}\right)\left(w_{4, n}-w_{2, n}\right)}{\left(w-w_{2, n}\right)\left(w_{4, n}-w_{3, n}\right)} .
$$

Then $\tilde{\phi}_{n}$ map $\Delta$ conformally onto $H$ taking $w_{1, n}, w_{2, n}, w_{3, n}, w_{4, n}$ onto $b_{n}, \infty, 0,1$ respectively. We also have $1<b_{n}<\infty$ and $b_{n} \rightarrow \infty$ as $n \rightarrow \infty$. Let $H(a, \infty, 0,1)$ be the quadrilateral with vertices $a, \infty, 0,1$ and domain $H$. If we set $(m(a))^{-1}=$ $M(H(a, \infty, 0,1)$, then we have

$$
\frac{M\left(f\left(Q_{n}\right)\right)}{M\left(Q_{n}\right)}=\frac{m\left(a_{n}\right)}{m\left(b_{n}\right)}
$$

and

$$
m(a)=\frac{K\left(\sqrt{1-\frac{1}{a}}\right)}{K\left(\frac{1}{\sqrt{a}}\right)}
$$

where

$$
K(t)=\int_{0}^{1} \frac{d x}{\sqrt{\left(1-x^{2}\right)\left(1-t^{2} x^{2}\right)}}
$$

(cf. [8, pp. 59-60] and [1]).

As $K(0)=\frac{\pi}{2}$ and

$$
K(t) \sim \frac{1}{2} \log \frac{1}{1-t} \quad \text { as } \quad t \rightarrow 1-,
$$


we have

$$
m(a) \sim \frac{1}{\pi} \log a, \quad \text { as } \quad a \rightarrow \infty .
$$

Therefore, when $n$ is sufficiently large, we have

$$
a_{n}=\left|a_{n}\right| \sim \frac{C_{1}}{\left|z_{1, n}-z_{2, n}\right|},
$$

and

$$
b_{n}=\left|b_{n}\right| \sim \frac{C_{2}}{\left|w_{1, n}-w_{2, n}\right|},
$$

where $C_{1}$ and $C_{2}$ are positive constants.

Recall that the local dilatation $H_{z_{1}}(f)$ (which can be defined similarily as the unit circle case) of $f$ is the infimum of the dilatations of possible extensions $\tilde{f}$ of $f$ to the neighborhoods of $z_{1}$. We shall prove that $K_{0}^{d}(f) \leq H_{z_{1}}(f)$.

Let $\varepsilon>0$ be arbitrarily given. Then there is a quasiconformal extension $\tilde{f}_{\varepsilon}$ of $f$ in a neighborhood $U_{\varepsilon}=\left\{z ;\left|z-z_{1}\right|<\varepsilon\right\}$ of $z_{1}$ with maximal dilatation $K\left(\tilde{f}_{\varepsilon}\right) \leq H_{z_{1}}(f)+\varepsilon$. From the basic properties of quasiconformal mappings, $\tilde{f}_{\varepsilon}$ is Hölder continuous with Hölder index $\frac{1}{K\left(\tilde{f}_{\varepsilon}\right)}$ and a coefficient depending on $U_{\varepsilon}$ and $f_{\varepsilon}$. We deduce that for all sufficiently large $n$

$$
\frac{1}{H_{z_{1}}(f)+\varepsilon^{\prime}} \leq \frac{\log \left|w_{n, 1}-w_{n, 2}\right|}{\log \left|z_{n, 1}-z_{n, 2}\right|} \leq H_{z_{1}}(f)+\varepsilon^{\prime}
$$

where $\varepsilon^{\prime} \rightarrow 0$ as $\varepsilon \rightarrow 0$. This implies

$$
\frac{1}{H_{z_{1}}(f)+\varepsilon^{\prime \prime}} \leq \frac{M\left(f\left(Q_{n}\right)\right)}{M\left(Q_{n}\right)} \leq H_{z_{1}}(f)+\varepsilon^{\prime \prime}
$$

for all sufficiently large $n$, where $\varepsilon^{\prime \prime} \rightarrow 0$ as $\varepsilon \rightarrow 0$.

Letting $n \rightarrow \infty$ and $\varepsilon \rightarrow 0$ and noting that $H_{z_{1}}(f) \leq H(f)$, we get the desired result in case (1).

\section{Case (2). A pair of two points degeneracy}

In this case we use similar transformations to obtain

$$
\begin{gathered}
a_{n}=\frac{\left(z_{1, n}-z_{3, n}\right)\left(z_{4, n}-z_{2, n}\right)}{\left(z_{1, n}-z_{2, n}\right)\left(z_{4, n}-z_{3, n}\right)} \sim \frac{C_{1}}{\left(z_{1, n}-z_{2, n}\right)\left(z_{4, n}-z_{3, n}\right)}, \\
b_{n}=\frac{\left(w_{1, n}-w_{3, n}\right)\left(w_{4, n}-w_{2, n}\right)}{\left(w_{1, n}-w_{2, n}\right)\left(w_{4, n}-w_{3, n}\right)} \sim \frac{C_{2}}{\left(w_{1, n}-w_{2, n}\right)\left(w_{4, n}-w_{3, n}\right)},
\end{gathered}
$$

where $C_{1}$ and $C_{2}$ are positive constants. 
Therefore we have

$$
\frac{\pi}{M\left(Q_{n}\right)} \sim \log a_{n} \sim-\log \left|z_{1, n}-z_{2, n}\right|-\log \left|z_{4, n}-z_{3, n}\right|
$$

and

$$
\frac{\pi}{M\left(f\left(Q_{n}\right)\right.} \sim \log b_{n} \sim-\log \left|w_{1, n}-w_{2, n}\right|-\log \left|w_{4, n}-w_{3, n}\right| .
$$

Now we perform the same procedure as in Case (1) to the neighborhoods of $z_{1}=z_{2}$ and $z_{3}=z_{4}$ respectively. We deduce that

$$
K_{0}^{d}(f) \leq \max \left\{H_{z_{1}}(f), H_{z_{3}}(f)\right\} \leq H(f)
$$

as required.

\section{Case (3). Three points degeneracy}

In this case, we set

$$
\phi_{n}(z)=e^{i \theta_{n}} \frac{z-z_{1, n}}{z-z_{4, n}},
$$

where $\theta_{n}$ is chosen so that $\phi_{n}$ maps $\Delta$ to $H$. Similarly let

$$
\tilde{\phi}_{n}(w)=e^{i \tilde{\theta}_{n}} \frac{w-f\left(z_{1, n}\right)}{w-f\left(z_{4, n}\right)}
$$

such that $\tilde{\phi}_{n}$ maps $\Delta$ to $H$. Thus, without loss of generality, We can use the upper half plane $H$ instead of $\Delta$ and assume that $\lim _{n \rightarrow \infty} z_{j, n}=z_{1} \in R,(j=1,2,3)$, $z_{4, n}=\infty$ and that $\lim _{n \rightarrow \infty} f\left(z_{j, n}\right)=f\left(z_{1}\right) \in R,(j=1,2,3), f\left(z_{4, n}\right)=\infty$.

For any given $\varepsilon>0$ we choose a quasiconformal extension $\tilde{f}_{\varepsilon}$ in $U_{\varepsilon}=\{z ; \mid z-$ $\left.z_{1} \mid<\varepsilon\right\}$ of $f$ such that the maximal dilatation of $\tilde{f}_{\varepsilon}$ in $U_{\varepsilon}$ is at most $H_{z_{1}}(f)+$ $\varepsilon$. From the theory of quasiconformal mappings it is possible to extend $\tilde{f}_{\varepsilon}$ to a quasiconformal mapping of the whole plane, which is still denoted by $\tilde{f}_{\varepsilon}$, with bounded dilatation (e.g., using Beurling-Ahlfors extensions (cf., [2])).

Let $\Lambda_{n}$ be the extremal length of the family of curves in $H$ which join the intervals $\left[z_{1, n}, z_{2, n}\right]$ to $\left[z_{3, n}, \infty\right]$. Let $\tilde{\Lambda}_{n}$ be the extremal length of the family of curves in $H$ which join the interval $\left[f\left(z_{1, n}\right), f\left(z_{2, n}\right)\right]$ to $\left[f\left(z_{3, n}\right), \infty\right]$. Then we have

$$
\frac{M\left(f\left(Q_{n}\right)\right.}{M\left(Q_{n}\right)}=\frac{\tilde{\Lambda}_{n}}{\Lambda_{n}} \rightarrow K_{0}^{d}(f) \quad\left(\text { or } \quad \frac{1}{K_{0}^{d}(f)}\right) .
$$

Grötzsch's length-area argument (cf. [5]) shows that

$$
\frac{\tilde{\Lambda}_{n}}{\Lambda_{n}} \leq \iint_{C} K\left(\tilde{f}_{\varepsilon}(z)\right)|\phi(z)| d x d y
$$


where

$$
\phi(z)=\frac{C\left(z_{1, n}, z_{2, n}, z_{3, n}\right)}{\left(z-z_{1, n}\right)\left(z-z_{2, n}\right)\left(z-z_{3, n}\right)}
$$

and where the constant $C\left(z_{1, n}, z_{2, n}, z_{3, n}\right)$ can be chosen to satisfy

$$
\iint_{C}|\phi(z)| d x d y=1 .
$$

Therefore $C\left(z_{1, n}, z_{2, n}, z_{3, n}\right) \rightarrow 0$ as $z_{j, n} \rightarrow z_{1}(n \rightarrow \infty, j=1,2,3)$.

As $C\left(z_{1, n}, z_{2, n}, z_{3, n}\right) \rightarrow 0$, the complement of $U_{\varepsilon}$ has arbitrarily small mass with respect to the measure of $|\phi(z)| d x d y$. Note that $K\left(\tilde{f}_{\varepsilon}\right)$ is uniformly bounded, we must have

$$
\frac{\tilde{\Lambda}_{n}}{\Lambda_{n}} \leq H_{z_{1}}(f)+2 \varepsilon
$$

for all sufficiently large $n$.

Letting $n \rightarrow \infty$ and $\varepsilon \rightarrow 0$, the proof of Case (3) is completed provided that $\frac{\tilde{\Lambda}_{n}}{\Lambda_{n}}=\frac{M\left(f\left(Q_{n}\right)\right.}{M\left(Q_{n}\right)}$. But the proof still works if $\frac{\Lambda_{n}}{\tilde{\Lambda}_{n}}=\frac{M\left(f\left(Q_{n}\right)\right)}{M\left(Q_{n}\right)}$, we only need to change the curve families. This completes the proof of Case (3).

\section{Case (4). Four points degeneracy}

We can treat this case similarly as we did in Case (3). We work on $H$ and, without loss of generality, assume that all points involved are finite.

Let $\Lambda_{n}$ be the extremal length of the family of curves in $D$ which join the intervals $\left[z_{1, n}, z_{2, n}\right]$ to $\left[z_{3, n}, z_{4, n}\right]$. Let $\tilde{\Lambda}_{n}$ be the extremal length of the family of curves in $H$ which join the interval $\left[f\left(z_{1, n}\right), f\left(z_{2, n}\right)\right]$ to $\left[f\left(z_{3, n}\right), f\left(z_{4, n}\right)\right]$. Then we have

$$
\frac{M\left(f\left(Q_{n}\right)\right.}{M\left(Q_{n}\right)}=\frac{\tilde{\Lambda}_{n}}{\Lambda_{n}} \rightarrow K_{0}^{d}(f) \quad\left(\text { or } \quad \frac{1}{K_{0}^{d}(f)}\right) .
$$

Use the same proof of Case (3) and note that the extremal holomorphic functions will be changed to

$$
\phi(z)=\frac{C\left(z_{1, n}, z_{2, n}, z_{3, n}, z_{4, n}\right)}{\left(z-z_{1, n}\right)\left(z-z_{2, n}\right)\left(z-z_{3, n}\right)\left(z-z_{4, n}\right)}
$$

where, again, the constant $C\left(z_{1, n}, z_{2, n}, z_{3, n}, z_{4, n}\right) \rightarrow 0$ as $z_{j, n} \rightarrow z_{1}(n \rightarrow \infty$, $j=1,2,3,4)$. Thus we can prove this case similarly as we did in Case (3).

The proof of Theorem 1 is completed. 


\section{$\S 3$. Proof of Theorem 2}

Assume that $f_{1}, f_{2} \in Q S(\Gamma)$ and there exist quadrilaterals $Q_{1}, Q_{2}$ with properties that

$$
M\left(Q_{1}\right)=M\left(Q_{2}\right)
$$

and

$$
K_{0}^{q}\left(f_{1}\right)=K_{1}\left(f_{1}\right)=K_{1}\left(f_{2}\right)=K_{0}^{q}\left(f_{2}\right)
$$

where

$$
K_{1}\left(f_{1}\right)=\frac{M\left(f_{1}\left(Q_{1}\right)\right)}{M\left(Q_{1}\right)}
$$

and

$$
K_{1}\left(f_{2}\right)=\frac{M\left(f_{2}\left(Q_{2}\right)\right)}{M\left(Q_{2}\right)} .
$$

We will show that $f_{1} \circ f_{2}^{-1} \in \operatorname{Möb}(\Gamma)$, that is, $\left[f_{1}\right]=\left[f_{2}\right]$.

To prove the fact above, we denote the rectangle with vertices $0, K, K+i, i$ by $\mathcal{R}(K)$, where $K>1$.

Now let $\phi_{1}$ and $\phi_{2}$ be the conformal mappings from $Q_{1}$ and $Q_{2}$ to the rectangle $\mathcal{R}\left(M\left(Q_{1}\right)\right)=\mathcal{R}\left(M\left(Q_{2}\right)\right)$ respectively, and let $\tilde{\phi}_{1}$ and $\tilde{\phi}_{2}$ be the conformal mappings from $f\left(Q_{1}\right)$ and $f\left(Q_{2}\right)$ to the rectangle $\mathcal{R}\left(M\left(f_{1}\left(Q_{1}\right)\right)\right)=\mathcal{R}\left(M\left(f_{2}\left(Q_{2}\right)\right)\right)$ respectively.

The only quasiconformal mapping from $\mathcal{R}\left(M\left(Q_{1}\right)\right)$ to $\mathcal{R}\left(M\left(f_{1}\left(Q_{1}\right)\right)\right)$ with dilatation $K=K_{0}\left(f_{1}\right)=\frac{M\left(f_{1}\left(Q_{1}\right)\right)}{M\left(Q_{1}\right)}$ is $f_{K}(x+i y)=K x+i y$ (cf. [8]). Therefore $f_{1}$ and $f_{2}$ have extremal quasiconformal extensions

$$
\tilde{f}_{1}=\tilde{\phi}_{1}^{-1} \circ f_{K} \circ \phi_{1} \quad \text { and } \quad \tilde{f}_{2}=\tilde{\phi}_{2}^{-1} \circ f_{K} \circ \phi_{2}
$$

respectively. Thus we have

$$
\tilde{f}_{1} \circ \tilde{f}_{2}^{-1}=\tilde{\phi}_{1}^{-1} \circ f_{K} \circ \phi_{1} \circ \phi_{2}^{-1} \circ f_{K}^{-1} \circ \tilde{\phi}_{2} .
$$

By computing the Beltrami coefficient of $\tilde{f}_{1} \circ \tilde{f}_{2}^{-1}$, we see that $\frac{\partial}{\partial \bar{z}}\left(\tilde{f}_{1} \circ \tilde{f}_{2}^{-1}\right)=0$. So $\tilde{f}_{1} \circ \tilde{f}_{2}^{-1}$ is a conformal mapping from $\Delta \rightarrow \Delta$, i.e., $f_{1} \circ f_{2}^{-1} \in \operatorname{Möb}(\Gamma)$.

The argument above shows that every $[f] \in U$ can be determined by the module of a quadrilateral and the dilatation of the extremal quasiconformal extension.

On the other hand, suppose that for $j=1,2, f_{j} \in Q S(\Gamma)$ satisfy

$$
K_{0}^{q}\left(f_{j}\right)=K_{1}\left(f_{j}\right)=\frac{M\left(f_{j}\left(Q_{j}\right)\right)}{M\left(Q_{j}\right)},
$$

where $Q_{j}$ are quadrilaterals. If $M\left(Q_{1}\right) \neq M\left(Q_{2}\right)$ or $K_{1}\left(f_{1}\right) \neq K_{1}\left(f_{2}\right)$, then $f_{1} \circ f_{2}^{-1} \notin P S L(2, R)$. This implies the first part of the theorem. 
We next show that every $[f] \in U$ is a Strebel point (this might be a known result, we include the simple proof here for the completeness of the paper).

From the argument above, for every $[f]$ there exist a quadrilateral $Q$ with domain $\Delta$ and a constant $K>1$ such that $f$ has a quasiconformal extension $\tilde{f}=(\tilde{\phi})^{-1} \circ f_{K} \circ \phi$, where $\phi: Q \rightarrow \mathcal{R}(M(Q)$ and $\tilde{\phi}: f(Q) \rightarrow \mathcal{R}(M(f(Q)))$ are conformal mappings and $f_{K}=K x+i y: \mathcal{R}(M(Q)) \rightarrow \mathcal{R}(M(f(Q)))$ is the affine stretch mapping. As the (local) dilatation of a quasiconformal mapping does not change if it composes a conformal mapping. So we can estimate the local dilatation (which can be defined similarly as the unit circle case ) of $f_{K}(z): \partial \mathcal{R}(M(Q)) \rightarrow$ $\partial \mathcal{R}(M(f(Q)))$.

Let $\xi \in \partial \mathcal{R}(M(Q))$. Suppose first that $\xi$ is not a vertex of $\partial \mathcal{R}(M(Q))$. Since the boundary correspondence in a neighborhood of $\xi$ is smooth, $H_{\xi}\left(f_{K}\right)=1$ (cf. [11]). We next suppose that $\xi$ is one of the four vertices of $\partial \mathcal{R}(M(Q))$. Note that the local dilatations of $f_{K}$ at the four vertices are the same (cf. [10]). Thus we may suppose $\xi=0$. Since in [10] it was proved that $f_{K}$ is not an extremal quasiconformal mapping from $\left\{z ; 0<\arg z<\frac{\pi}{2}\right\} \rightarrow\left\{z ; 0<\arg z<\frac{\pi}{2}\right\}$, this implies $H_{\xi}\left(f_{K}\right)<K=K_{1}(f)$. The proof of the theorem is completed.

\section{$\S 4$. Proof of Theorem 4}

Since $H(f)=1$ for every $f \in[\tilde{f}] \in \mathcal{T}_{0}$, we must have $K_{0}(f)=K_{0}^{q}(f)$ for $f \notin$ $\operatorname{Möb}(\Gamma)$. To prove the theorem, we only need to prove that every $f \in[\tilde{f}] \in U \backslash\{[i d]\}$ has the property that $K_{0}^{q}(f) \neq K_{1}(f)$.

Now assume, for the contrary, $f \in[\tilde{f}] \in U \backslash\{i d\}$. Then $K_{0}^{q}(f)=K_{1}(f)$. Denote $K=K_{1}(f)$. We shall prove the following fact that there is a point $z_{0} \in \Gamma$ at which the local quasisymmetric constant of $f$ is $K^{2}$.

In the following we use the upper plane again. Suppose that $z_{1}, z_{2}, z_{3}, z_{4} \in R$ follow each other in the positive (anticlockwise) direction on $R$. We still denote the quadrilateral with domain $H$ and vertices $z_{1}, z_{2}, z_{3}, z_{4}$ by $Q=Q\left(z_{1}, z_{2}, z_{3}, z_{4}\right)$. Now assume that $f(Q)=Q\left(f\left(z_{1}\right), f\left(z_{2}\right), f\left(z_{3}\right), f\left(z_{4}\right)\right)$ such that $K_{0}^{q}(f)=\frac{M(f(Q))}{M(Q)}$ $=K_{1}(f)$.

Let $\phi$ and $\tilde{\phi}$ be the conformal mappings such that $\phi(Q)=\mathcal{R}(M(Q))$ and $\tilde{\phi}(f(Q))=\mathcal{R}(M(f(Q))$. As before the unique extremal quasiconformal mapping from $\mathcal{R}(M(Q))_{\text {c }}$ to $\mathcal{R}(M(f(Q)))$ is $f_{K}(x+i y)=K x+i y$. Now suppose that $\phi\left(z_{1}\right)=0$ and $\tilde{\phi}\left(f\left(z_{1}\right)\right)=0$. From the classical elliptic integral theory, we have

$$
\begin{aligned}
& \phi(z)=\left(z-z_{1}\right)^{\frac{1}{2}}\left\{a_{0}+a_{1}\left(z-z_{1}\right)+a_{2}\left(z-z_{1}\right)^{2}+\ldots\right\} \\
= & a_{0}\left(z-z_{1}\right)^{\frac{1}{2}}+O\left(\left(z-z_{1}\right)^{\frac{3}{2}}\right) \quad\left(z \in R \quad \text { and } \quad z \rightarrow z_{1}\right),
\end{aligned}
$$

and

$$
\tilde{\phi}\left(f\left(z_{1}\right)\right)=\tilde{a}_{0}\left(w-f\left(z_{1}\right)\right)^{\frac{1}{2}}+O\left(\left(w-f\left(z_{1}\right)\right)^{\frac{3}{2}}\right) \quad\left(w \in R \quad \text { and } \quad w \rightarrow f\left(z_{1}\right)\right) .
$$


Since $f=\tilde{\phi}^{-1} \circ f_{K} \circ \phi$, we have locally

$$
f(z)= \begin{cases}C K^{2}\left(z-z_{1}\right)+o\left(z-z_{1}\right) & z>z_{1}, \\ C\left(z-z_{1}\right)+o\left(z-z_{1}\right) & z \leq z_{1},\end{cases}
$$

where $z \in R$ and $C \neq 0$ is a constant. This implies

$$
\lim _{t \rightarrow 0^{+}} \frac{f\left(z_{1}+t\right)-f\left(z_{1}\right)}{f\left(z_{1}\right)-f\left(z_{1}-t\right)}=K^{2} .
$$

On the other hand it was proved in [6] (also cf. [11]) that if $f \in[\tilde{f}] \in \mathcal{T}_{0}$, the local quasisymmetric constant above must be equal to 1 . This contradiction proves Theorem 4 .

\section{$\S 5$. Affine stretch mappings}

In [1], the following result is proved.

Theorem A. For each $K>1$, there exists a sense-preserving quasisymmetric homeomorphism $f$ of $\Gamma$ such that

$$
K_{0}(f)<K_{1}(f)=K .
$$

To prove the theorem, the authors constructed concrete quasiconformal mappings as follows. Let $V$ be the closed parallelogram with vertices $\xi_{1}=0, \xi_{2}=1$ $\xi_{3}=\alpha+1+i \beta, \xi_{4}=\alpha+i \beta$, where $\alpha>0$ and $\beta>0$. Let $f_{K}(V)$ be the image of $V$ under the horizontal affine stretch $f_{K}$ that takes $x+i y$ onto $K x+i y$ so that the vertices of $f_{K}(V)$ are $\tilde{\xi}_{1}=0, \tilde{\xi}_{2}=K, \tilde{\xi}_{3}=K(\alpha+1)+i \beta, \tilde{\xi}_{4}=K \alpha+i \beta$. Let $\phi$ and $\tilde{\phi}$ be the conformal mappings from $V$ and $f_{K}(V)$ to $\Delta$, respectively. Since $f_{K}$ is uniquely extremal for its boundary values, the mapping $\tilde{f}_{K}=\tilde{\phi} \circ f_{k} \circ \phi^{-1}$ of $\Delta$ onto $\Delta$ is uniquely extremal for its boundary values. Under this construction, we see easily that any internal angle with vertex at one of $\xi_{j}$ and $\tilde{\xi}_{j}(\mathrm{j}=1,2,3,4)$ cannot be equal to $\frac{\pi}{2}$. It was proved in [1] that $\tilde{f}_{K}$ has the property that $K_{0}\left(\tilde{f}_{K}\right)<K_{1}\left(\tilde{f}_{K}\right)$.

Now we, by using our results, give a simpler proof of Theorem A.

\section{Proof of Theorem A}

In fact we can prove that $\left[\tilde{f}_{K}\right] \notin U$ and $\left[\tilde{f}_{K}\right] \in \mathcal{T}_{S}$. Therefore Theorem A follows from Theorem 3.

The proof of $\left[\tilde{f}_{K}\right] \notin U$ is simple and we omit it (cf. [1]).

Now we show $H\left(\tilde{f}_{K}\right)<K=K_{1}\left(\left[\tilde{f}_{K}\right]\right)$ (this is the main part of [1]). From Fehlmann's result (cf. [4] and [11]) and $H_{\xi}\left(f_{K}\right)=1$ for all $\xi \in \partial V$ and $\xi \neq \xi_{j}(j=$ 
$1,2,3,4)$, we see that $H\left(\tilde{f}_{K}\right)=H_{\xi_{j}}\left(f_{K}\right)$ for some $j=1,2,3,4$. So we need to show $H_{\xi_{j}}\left(f_{K}\right)<K$ for $j=1,2,3,4$. Since at each vertex $\xi_{j}, f_{K}$ is the restriction of the same affine stretch on an angular domain whose vertex is $\xi_{j}$ and whose boundary is the extension of two sides of the parallelogram, it is known that $f_{K}$ is not extremal for its boundary values (cf. [9] and [10]). (This is a known result if the vertex of an angular domain is the origin. Note that for the case of affine stretch mappings, the extremal problem for the boundary values of an angular domain depends only on the the family of holomorphic functions defined on the domain (cf. [9]), it is easy to see that the affine stretch mappings cannot be extremal for the boundary values of an angular domain whether or not the vertex of it is the origin.) Therefore there is a quasiconformal mapping $F$ of dilatation $<K$ with the same boundary value of $f_{K}$ on the two sides of the parellelogram. This implies $H_{\xi_{j}}\left(f_{K}\right)<K(j=1,2,3,4$.$) . This completes the proof of Theorem A.$

\section{References}

[1] Anderson J.M. and Hinkkanen A., Quadrilaterals and extremal quasiconformal extensions, Comment. Math. Helvetici 70 (1995), 455-474.

[2] Beurling A. and Ahlfors L. V., The boundary correspondence under quasiconformal mappings, Acta Math. 96 (1956), 125-142.

[3] Earle C. J. and Li Z., Isometrically embedded polydisks in infinite dimensional Teichmüller spaces, J. of Geometric Analyses, to appear.

[4] Fehlmann, R., Quasiconformal mappings with free boundary components, Ann. Acad. Sci. Fenn. 7(2) (1982), 337-347.

[5] Gardiner F. P., Teichmüller theory and quadratic differentials, Wiley-Interscience, New York 1987.

[6] Gardiner F. P. and Sullivan D., Symmetric structures on a closed curve, Amer. J. Math. 114 (1992), 683-736.

[7] Gardiner F. P. and Sullivan D., Lacunary series as quadratic differentials in conformal dynamics, Contemporary Math. 169, AMS., Providence, RI, (1994), 307-330.

[8] Lehto O. and Virtanen K. I., Quasiconformal mappings in the plane, Springer, Berlin 1973.

[9] Reich E., Quasiconformal mappins of the disk with given boundary values, Lecture Notes in Mathematics, Springer, Berlin 505 (1976), 101-137.

[10] Strebel K., Zur frage der Eindentigkeit extremaler quasikoformer Abbildungen des Einheitskreises, Comment.Math. Helvetici 36 (1962), 306-323.

[11] Strebel, K., On the existence of extremal Teichmüller mappins, J. Anal. Math. 30 (1976), 464-480.

[12] Yang S., Extremal quasiconformal extensions, preprint, 1993.

Shengjian Wu

Department of Mathematics

Peking University

Beijing 100871

P.R.China

e-mail: wusj@pku.edu.cn

(Received: December 8, 1996) 